\title{
Hedgehog signaling regulates the expression levels of inflammatory mediators in cigarette-induced airway inflammation
}

\author{
YI GUO, GUOCHAO SHI, HUANYING WAN and MIN ZHOU \\ Department of Pulmonary and Critical Care Medicine, Shanghai Rui Jin Hospital, School of Medicine, \\ Shanghai Jiao Tong University, Shanghai 200025, P.R. China
}

Received October 22, 2017; Accepted March 27, 2018

DOI: $10.3892 / \mathrm{mmr} .2018 .8861$

\begin{abstract}
Chronic obstructive pulmonary disease (COPD) is a persistent airway inflammation influenced by cigarette smoke. Previous studies have reported that Hedgehog (Hh) signaling is aberrantly activated by cigarette smoke and dysregulated in COPD. The present study explored the role of the $\mathrm{Hh}$ signaling pathway on the expression levels of certain inflammatory mediators in cigarette-induced airway inflammation. Herein, a total of three A549 cell populations were generated: The A0 group as control cells, the A1 group cells treated with nicotine at a concentration of $10 \mu \mathrm{M}$ for 12,24 and $48 \mathrm{~h}$, and the A2 group cultured simultaneously with nicotine and cyclopamine for the same duration. The total concentrations of the inflammatory mediators interleukin-6 (IL-6), IL-8 and tumor necrosis factor (TNF)- $\alpha$, and an anti-inflammatory cytokine, IL-10, were assessed in all of the cells by ELISA and western blotting. The protein levels of sonic hedgehog (Shh), glioma-associated oncoprotein 1 (Gli1) and Smoothened (Smo) in nicotine-induced $\mathrm{Hh}$ signaling were also detected. The results indicated that A549 had increased levels of IL-6, IL- 8 and TNF- $\alpha$ when cultured with nicotine when compared with the control cells. By contrast, the expression levels of these inflammatory mediators decreased with varying degrees when treated with cyclopamine that blocked the Hh signaling pathway. The IL-10 expression levels exhibited the reverse. The expressions of the Shh, Gli1 and Smo proteins were higher in the A1 group when compared with the control and decreased with cyclpoamine treatment. In conclusion, the Hh signaling
\end{abstract}

Correspondence to: Professor Min Zhou, Department of Pulmonary and Critical Care Medicine, Shanghai Rui Jin Hospital, School of Medicine, Shanghai Jiao Tong University, 197 Road Rui Jin Er, Shanghai 200025, P.R. China

E-mail: doctor_zhou_99@163.com

Abbreviations: COPD, chronic obstructive pulmonary disease; GWAS, genome-wide association study; Hh, Hedgehog; HIP, edgehog interacting protein; SNP, single-nucleotide polymorphisms

Key words: cigarette-induced airway inflammation, Hedgehog signaling, COPD, inflammatory mediator pathway may partly have an impact on cigarette-induced airway inflammation via the regulation of inflammatory mediators. Thus, blocking Hh signaling and diminishing the airway inflammation reaction may serve as a potential therapy for COPD.

\section{Introduction}

Chronic obstructive pulmonary disease (COPD) is mainly a consequence of persistent airway inflammation caused by cigarette smoke (CS) and genetic predisposition (1). The exposure to cigarette smoke (CS) activates the hedgehog (Hh) signaling pathway in bronchial epithelial cells. Hh activation occurs in BEAS2B cells when repeatedly exposed to smoke (2).

Hh signaling is one of the most conserved pathways, that regulates morphogenesis of multiple organs, including lung, intestines, and nervous system during embryogenesis. In the adult, Hh signaling also maintains organ homeostasis and modulates tissue repair (3). The Hh pathway is comprised of a complex network of molecules. Three Hh ligands have been identified: sonic hedgehog (Shh) for human, Indian hedgehog (Ihh) and desert hedgehog (Dhh). Hh ligands bind to their repressive receptor, Patched (Ptc), a twelve-transmembrane protein; it relieves the suppression of the signaling transducer, Smoothened (Smo), a seven-transmembrane protein, thereby activating the Hh pathway. The unleashed Smo passages from the vesicles to the primary cilium on the cell membrane, leading to translocation of the transcription factors and glioma-associated oncoproteins (Gli1, Gli2, Gli3), especially Gli1, and subsequently promotes the transcription of the target genes.

The first identified Hh blocker is cyclopamine. It is a steroid alkaloid and inhibits the Hh pathway by directly binding Smo, which causes developmental abnormalities in animals (4).

Previous data implicated a role of $\mathrm{Hh}$ in postnatal lung development; the interruption of $\mathrm{Hh}$ signaling at earlier time points leads to enlarged alveolar airspaces. In the adult lung, the Hh pathway maintains mesenchymal quiescence and is dysregulated in disease such as COPD (5). These results suggest that the $\mathrm{Hh}$ pathways underlie the initiation, maintenance, proliferation, and survival of CSE-transformed epithelial cells. However, the role of Hh signaling pathway in cigarette-induced airway inflammation remains unclear. 


\section{Materials and methods}

Cell culture and reagents. Human alveolar epithelial cells (A549) were purchased from the Shanghai Institutes for Biological Sciences and cultured in a humidified incubator with $5 \% \mathrm{CO}_{2}$ at $37^{\circ} \mathrm{C}$ in RPMI 1640 medium (Gibco Life Technologies, Gaithersburg, MD, USA) supplemented with $10 \%$ fetal bovine serum (FBS), penicillin, and streptomycin. After reaching early confluency, the cells were trypsinized and plated for experiments.

We generated three A549 cell populations. We defined the primary cultures as untreated control cells (A0 group), A1 group represented A549 cells stimulated with nicotine (Sigma-Aldrich St. Louis, MO, USA) at appropriate concentrations evaluated by Cell Counting Kit-8 (CCK-8; Dojindo Laboratories, Kumamoto, Japan) assay for 12, 24 and 48 h, and A2 group represented A549 cells treated simultaneously with nicotine and cyclopamine (Sigma-Aldrich) at a concentration of $10 \mu \mathrm{M}$ according to published studies (6-8).

Cell viability assay. The proliferation A549 cells was measured using the CCK-8 according to the manufacturer's instructions in order to determine the appropriate concentrations of nicotine treatment in the experiment. Cells $(2,000 / 100 \mu \mathrm{l} / \mathrm{well})$ were plated in triplicate into 96 -well plates and cultured before treatment with nicotine at $0.1,1,10$, and $100 \mu \mathrm{M}$ for 12,24 and $48 \mathrm{~h}$, respectively. Subsequently, the cells were incubated with CCK-8 reagent (10 $\mu \mathrm{l})$ for an additional $2 \mathrm{~h}$. The absorbance was determined at $450 \mathrm{~nm}$, and the cell viability was calculated as a percentage of the OD value in the control cells. The most appropriate concentration of nicotine was determined and used in downstream experiments.

ELISA. The total concentrations of specific inflammatory mediators (IL-6, IL-8, TNF- $\alpha$ ) in A0, A1 and A2 cells stimulated by nicotine with and without cyclopamine (at a concentration of $10 \mu \mathrm{M}$ ) were assessed by commercial ELISA kits (Anogen, Canada), according to the manufacturer's instructions. The total concentration of IL-10, known as the cytokine synthesis inhibitory factor (CSIF), which inhibits the production of IL-6, IL-8, TNF- $\alpha$ and downregulates inflammation was also measured by ELISA immunoassay. The results were expressed as pictogram of cytokine per milliliter plasma (pg/ml).

Western blot analysis. The expression levels of Shh, Smo, and Gli1 proteins in control A0, in A1 cells treated with $10 \mu \mathrm{M}$ nicotine, and in A2 cells cultured simultaneously with nicotine $(10 \mu \mathrm{M})$ and cyclopamine $(10 \mu \mathrm{M})$ for 24 and $48 \mathrm{~h}$ were assessed respectively using Western blot. We also measured the expressions of IL-6, IL-8, and TNF- $\alpha$ at protein levels in $\mathrm{A} 0, \mathrm{~A} 1$, and $\mathrm{A} 2$ groups at 12,24 and $48 \mathrm{~h}$ using the same methods. The cells were lysed in RIPA lysis buffer, and the lysates incubated on ice and centrifuged at $14,000 \mathrm{rpm}, 4^{\circ} \mathrm{C}$ for $15 \mathrm{~min}$. Total protein concentrations were determined by Bradford assay. The protein samples were subjected to SDS-PAGE and transferred to polyvinyliene difluoride membranes. The non-specific binding sites on the membranes were blocked with skimmed milk for $1 \mathrm{~h}$, and the blots were incubated with primary antibody overnight at $4^{\circ} \mathrm{C}(1: 1,000$,

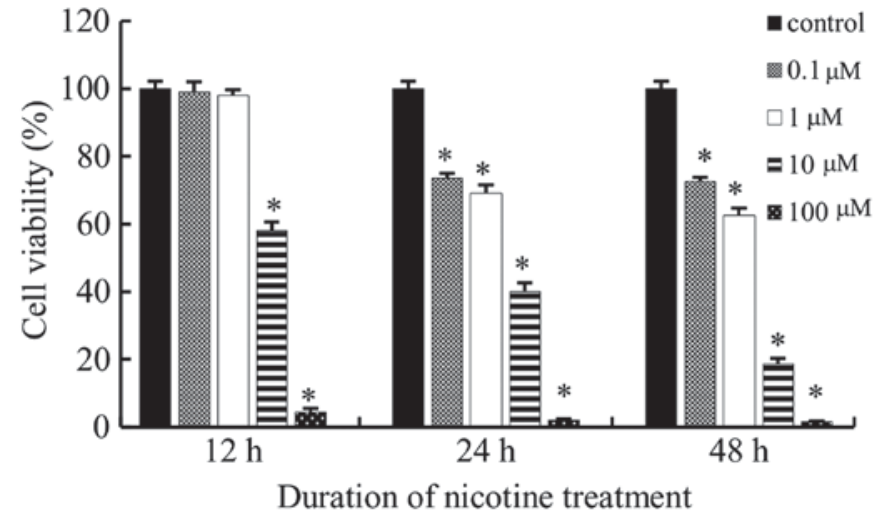

Figure 1. Cell viability of A549 cells treated with nicotine at different concentrations. ${ }^{*} \mathrm{P}<0.05$ vs. control.

Proteinch, USA). Subsequently, the membranes were probed with secondary antibodies (1:2,000, Santa Cruz, USA) for $1 \mathrm{~h}$ at room temperature. Finally, the relative protein levels were determined with Image $\mathbf{J}$ software.

Statistical analysis. All statistical analyses were performed using SPSS (version 20.0; IBM Corp., Armonk, NY, USA). The values are expressed as the mean \pm standard deviation. Analysis of variance was performed to evaluate the differences between the groups, followed by Scheffe post hoc test for multiple comparisons. A P-value $<0.05$ was considered as statistically significant.

\section{Results}

Cell viability assay. Cell viability curves were plotted as viable cell percentage based on the CCK-8 assay. As shown in Fig. 1, nicotine inhibited the proliferation of the tested cell lines in a time and dose dependent manner. According to the results of the CCK-8 assay, with nicotine treatment, a concentration of 0.1 and $1 \mu \mathrm{M}$ were not sufficient to stimulate cells at all the three time points; however, cells failed to survive at $100 \mu \mathrm{M}$. Therefore, $10 \mu \mathrm{M}$ was the most appropriate nicotine concentration used in the subsequent study.

Expression levels of $I L-6, I L-8, T N F-\alpha$, and $I L-10$ in cells by ELISA. A0 were cultured as control cells, A1 cells were stimulated with nicotine at a concentration of $10 \mu \mathrm{M}$ for 12,24 and $48 \mathrm{~h}$, respectively, and A2 cells were treated simultaneously with nicotine $(10 \mu \mathrm{M})$ and cyclopamine $(10 \mu \mathrm{M})$ for 12,24 and $48 \mathrm{~h}$, respectively. Nicotine induced the production of the inflammatory mediators in A549 cells.

We compared the IL-6 levels produced by A549 cells among the three groups. IL- 6 levels in A1 cells were increased significantly as compared to A0 cells at 24 and $48 \mathrm{~h}$ (all $\mathrm{P}<0.05$ ) but not at $12 \mathrm{~h}$. Supposedly, the $12 \mathrm{~h}$ time point was extremely short to induce the secretion of IL-6. Additionally, the levels of IL-6 in A2 group were significantly lower than A1 group at 24 and $48 \mathrm{~h}$ (all $\mathrm{P}<0.05$ ) (Fig. 2A).

The levels of IL-8 also increased significantly in A1 cells at 24 and $48 \mathrm{~h}$ as compared to the $\mathrm{A} 0$ group (all $\mathrm{P}<0.05$ ). The levels of IL-8 in the A2 group were significantly lower than that in the A1 group (12, 24, 48 h) (all $\mathrm{P}<0.05)$ (Fig. 2B). 

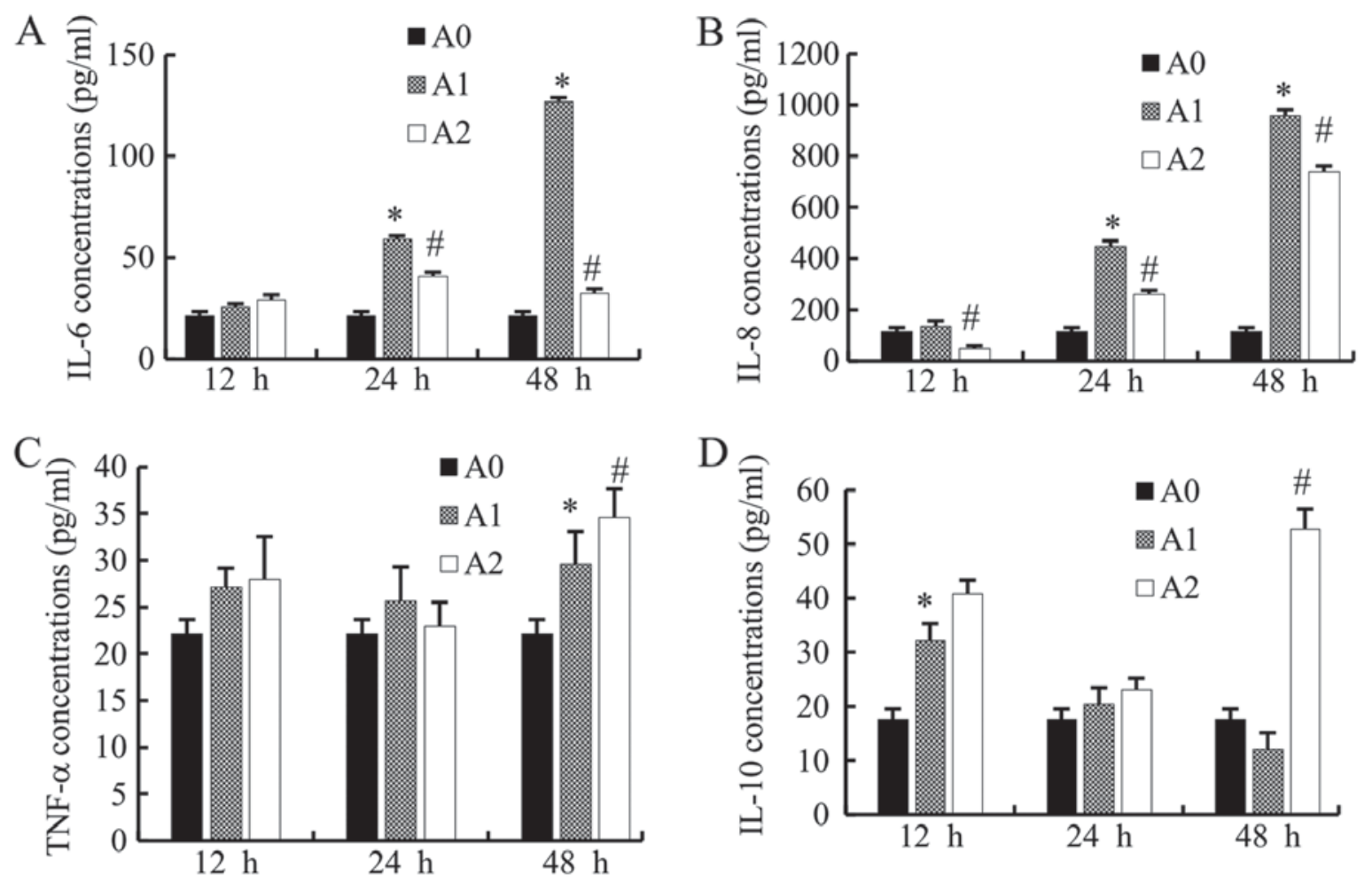

\section{Duration of nicotine treatment}

Figure 2. Concentrations of the inflammatory mediators in the A0, A1 and A2 groups by ELISA. The average concentrations of (A) IL-6, (B) IL-8, (C) TNF- $\alpha$ and (D) IL-10. ${ }^{~} \mathrm{P}<0.05$ vs. A0; ${ }^{*} \mathrm{P}<0.05$ vs. A1. IL, interleukin; TNF, tumor necrosis factor.

The TNF- $\alpha$ levels in A1 group were significantly higher than that in $\mathrm{A} 0$ at $48 \mathrm{~h}(\mathrm{P}<0.05)$; however, that in $\mathrm{A} 2$ decreased non-significantly as compared to A1 when treated simultaneously with cyclopamine only at $24 \mathrm{~h}$, while increased at 12 and $48 \mathrm{~h}(\mathrm{P}<0.05$ at $48 \mathrm{~h})$ (Fig. 2C). Therefore, the variation trend of TNF- $\alpha$ levels appears to be different from that of IL- 6 and IL-8. The underlying mechanism necessitates further investigation.

The level of the anti-inflammatory mediator, IL-10 in A1 group, increased at 12 and $24 \mathrm{~h}$ as compared to the control cells $(\mathrm{P}<0.05$ at $12 \mathrm{~h})$, while a decreased level was observed at $48 \mathrm{~h}$. When treated with cyclopamine, in contrary to IL-6 and IL-8, the IL-10 levels increased in A2 cells as compared to the A1 cells at $12,24,48 \mathrm{~h}$, but significantly at $48 \mathrm{~h}(\mathrm{P}<0.05)$ (Fig. 2D).

Expression levels of Shh, Gli1 and Smo proteins. We detected the faint expression of Shh protein in control cells (A0); however, when treated with nicotine (A1) at a concentration of $10 \mu \mathrm{M}$ for 24 and $48 \mathrm{~h}$, the levels of Shh increased significantly $(\mathrm{P}<0.05$ at 24 and $48 \mathrm{~h})$, while it decreased significantly with cyclopamine (A2) as compared to the A1 cells (Fig. 3). Since the expression of Gli1 reflects the activation of Hh pathway, we also measured the Glil levels. The results were similar to that of Shh. The expression of the Gli1 protein, stimulated by nicotine $(10 \mu \mathrm{M})$, was significantly higher than the control but decreased significantly with cyclopamine treatment for $48 \mathrm{~h}$ $(\mathrm{P}<0.05)$ (Fig. 3). The results of Smo protein were also similar to that of Shh, the expressions were significantly higher in A1 group than A0, but significantly lower in A2 compared to A1

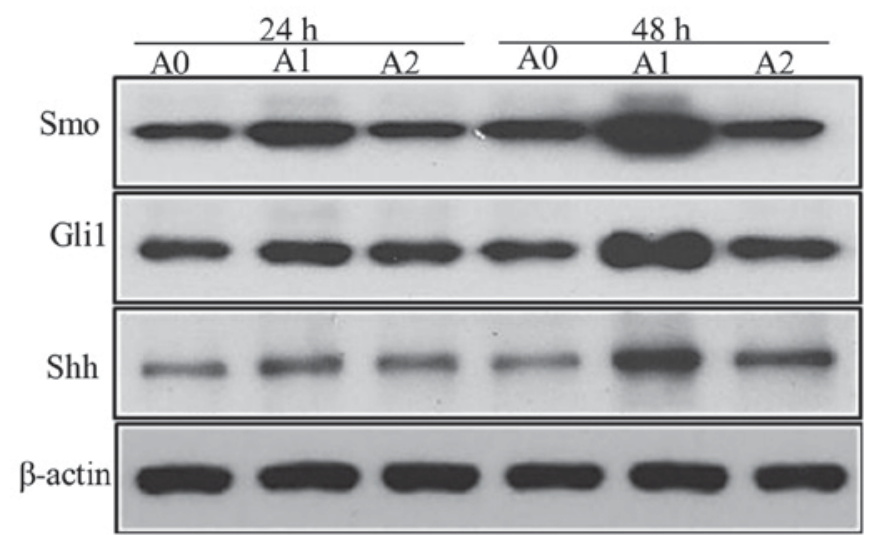

Figure 3. Shh, Gli1 and Smo expressions at the protein level in control cells (A0), in cells treated with $10 \mu \mathrm{M}$ nicotine (A1) and in cells treated with nicotine and cyclopamine (10 $\mu \mathrm{M}$; A2) for 24 and $48 \mathrm{~h}$, as determined by western blot analysis. Shh, sonic hedgehog; Gli1, glioma-associated oncoprotein 1; Smo, Smoothened.

$(\mathrm{P}<0.05$ at 24 and 48 h) (Fig. 3). The densitometric analyses of the three proteins are shown in Fig. 4.

Expressions of IL-6,IL-8,TNF- $\alpha$ at protein levels. We assessed the protein expressions of IL-6, IL-8, and TNF- $\alpha$ in A0, A1 and $\mathrm{A} 2$ cells using Western blot. We compared the expression levels of these inflammatory mediators produced by A549 cells between the three groups and found that the expression of IL-6 protein level in A1 cells increased compared to A0 at all the times, but significantly at $24 \mathrm{~h}(\mathrm{P}<0.05)$. On the other hand, 

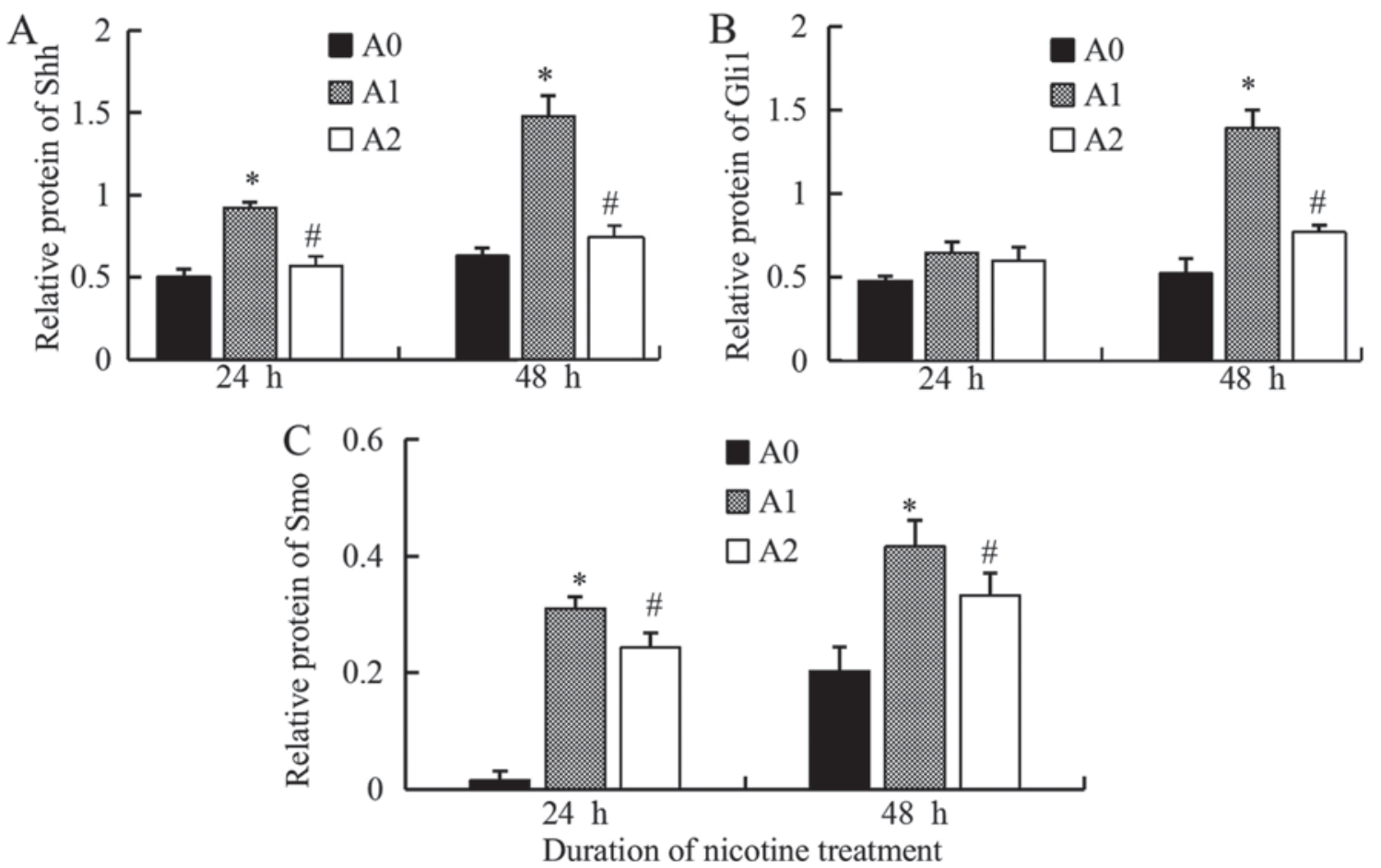

Figure 4. Relative protein levels of (A) Shh, (B) Gli1 and (C) Smo in A549 cells. "P<0.05 vs. A0; "P<0.05 vs. A1. Shh, sonic hedgehog; Gli1, glioma-associated oncoprotein 1; Smo, Smoothened.

the treatment with cyclopamine decreased the IL-6 level in the A2 group as compared to A1, the significant decrease was observed only at $24 \mathrm{~h}(\mathrm{P}<0.05)$ (Fig. 5). We suggest that $24 \mathrm{~h}$ is the most appropriate times for change of IL- 6 in inflammatory stimuli in the current study.

The IL-8 protein level in the A1 group was significantly higher than that for $\mathrm{A} 0$ group at 12,24 , and $48 \mathrm{~h}$ (all $\mathrm{P}<0.05$ ). The level was decreased with cyclopamine treatment in the A2 group, significantly at $48 \mathrm{~h}(\mathrm{P}<0.05)$ (Fig. 5). We suggest that $48 \mathrm{~h}$ is the most appropriate times for change of IL- 8 in inflammatory stimuli in the current study on Hh signaling.

The results of TNF- $\alpha$ protein were similar to that of IL-8; the level was maximal in A1 cells (all $\mathrm{P}<0.05)$ and decreased significantly in A2 cells as compared to A1 at $48 \mathrm{~h}(\mathrm{P}<0.05)$. Although these Western blot results were not completely identical as those of ELISA owing to the differences in the techniques, the results showed that the expression levels of the inflammatory mediators tended to decrease with cyclopamine treatment, when the Hh pathway was blocked (Fig. 5). The results of the densitometric analyses are shown in Fig. 6.

\section{Discussion}

In the current study, we measured the expression levels of some airway inflammatory mediators, such as IL-6, IL-8, TNF- $\alpha$ and also an anti-inflammatory mediator, IL-10, produced by A549 cells treated by nicotine with and without cyclopamine, the inhibitor of Hh pathway. Next, we attempted to explore the effect of $\mathrm{Hh}$ signaling on cigarette-induced airway inflammation, since few studies focusing on the role of such chronic airway disease were carried out.

The Hh pathway regulates organ homeostasis and stem cell renewal in the adult (9). This signaling is involved in early

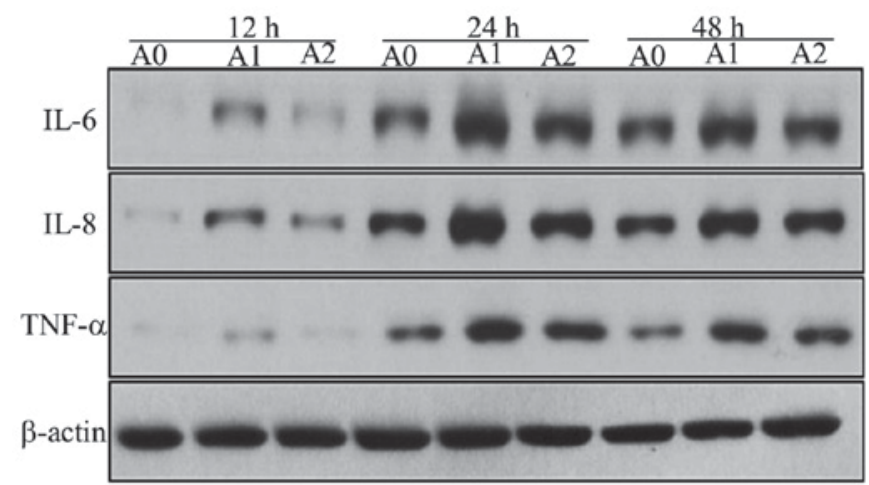

Figure 5. Expressions of IL-6, IL-8, and TNF- $\alpha$ at the protein level in A549 cells, as determined by western blot analysis. IL, interleukin; TNF, tumor necrosis factor.

embryogenesis, where it promotes cell growth, differentiation, tissue patterning, and vascularization. The canonical process of this pathway is that when Shh is secreted and reaches its target cells, Smo is suppressed, which results in the transcription of Gli1, Gli2, and Gli3. Nonetheless, Gli1 exclusively acts as a transcription activator, while Gli2 and Gli3 act either as repressors or activators $(10,11)$. In the nucleus, Gli1 modulates the transcription of a multitude of genes including Fox, Myc and cyclin $D$, involved in tissue development, differentiation, epithelial-mesenchymal transition (EMT), and stem cell maintenance.

Although the expression level of Shh is decreased at birth, it is continually observed in epithelial cells. The Hh pathway is also demonstrated to be involved in postnatal lung maturation (12). In the healthy adult lung, Hh signaling maintains adult lung quiescence and regulates repair (13). The Shh 

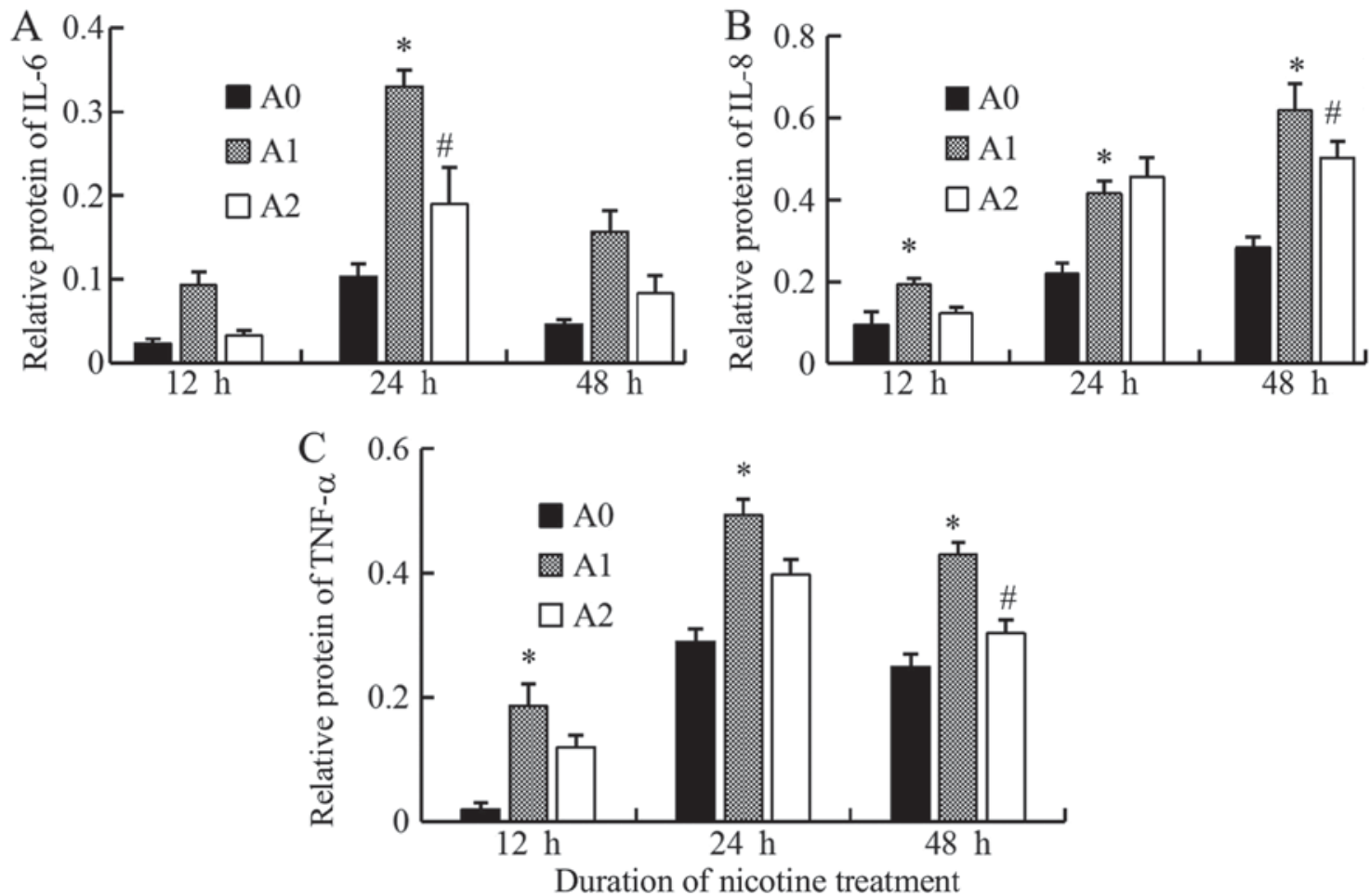

Figure 6. Relative protein levels of IL-6, IL-8, and TNF- $\alpha$ in A549 cells. (A) IL-6, (B) IL-8 and (C) TNF- $\alpha$. " P<0.05 vs. A0; "P<0.05 vs. A1. IL, interleukin; $\mathrm{TNF}$, tumor necrosis factor.

expression from the embryonic day is critical for branching and growing bronchi. The interruption of Hh signaling at earlier time points leads to defects in the wall structure of enlarged alveolar airspaces and fewer secondary septa (5), as well as, in the regeneration and repair after injury to adult lungs (10).

Some previous studies revealed that $\mathrm{Hh}$ signaling pathway was dysregulated in diseases such as COPD and idiopathic pulmonary fibrosis. The results of the study by Lemjabbar-Alaoui et al (2) demonstrated that CSE exposure activated Hh signaling in bronchial epithelial cells, rendering that $\mathrm{Hh}$ plays a causal role in the initiation, maintenance, proliferation, and survival of CSE-transformed epithelial cells; however, its role in airway inflammation is yet unclear.

The present study detected low Shh protein expression in A0 control cells; however, the levels increased significantly when treated with nicotine, and inhibited by cyclopamine. This phenomenon demonstrated that Hh signaling is lowly expressed in normal alveolar epithelial cells. We have also shown that exposure to nicotine results in aberrant reactivation of Hh pathway, as indicated by the marked expression of Shh. In addition, the expression level of Gli1 reflects the activation of Hh pathway significantly higher than control when stimulated by nicotine; however, it was decreased significantly with cyclopamine treatment. The results of Smo protein were similar to that of Gli1. Taken together, these results suggested that Hh signaling might be implicated in cigarette-induced airway diseases; nonetheless, the mechanism should be investigated further.

Systemic oxidative stress and inflammation have been implicated in COPD. This process involves the pro-inflammatory cytokines such as IL-6, IL-8, and TNF- $\alpha$, as the main promoters of the inflammation, which serve as markers of COPD progression. Therefore, we measured the levels of IL-6, IL-8, and TNF- $\alpha$ in A549 cells treated with nicotine and with and without cyclopamine using ELISA and Western blot. The alveolar epithelial cells distinctly produced higher levels of IL-6, IL-8, and TNF- $\alpha$ when cultured with nicotine as compared to control cells for 24 and $48 \mathrm{~h}$. Although Western blot results were not completely identical to that of ELISA; the results showed that the expression levels of these inflammatory mediators in A2 group tended to decrease with cyclopamine treatment at different time points as compared to the A1 group, IL-6 at $24 \mathrm{~h}, \mathrm{IL}-8$ at $48 \mathrm{~h}$, and TNF- $\alpha$ at $48 \mathrm{~h}$, significantly respectively). These results showed a potential role of Hh probably in cigarette-induced airway inflammation via regulation of specific inflammatory mediators' expressions. Hence, we suggested that smoking aberrantly reactivated the Hh pathway, while IL-6, IL-8, and TNF- $\alpha$ levels increase progressively, which partly enhances the airway inflammation. If the Hh signaling can be blocked, the expressions of these mediators may decrease, thereby reducing the inflammatory reaction.

Moreover, IL-10 was also tested in our study, along with the effect of $\mathrm{Hh}$ on an anti-inflammatory mediator. Its expression in $\mathrm{A} 1$ group was significantly higher than that in $\mathrm{A} 0$ at the $12 \mathrm{~h}$ time point, while a decrease was noted at $48 \mathrm{~h}$. Moreover, the IL-10 levels increased significantly in cells as a result of cyclopamine treatment at $48 \mathrm{~h}$, which was contrary to that of IL-6, IL-8, and TNF- $\alpha$. This phenomenon could be explained by its role of anti-inflammation.

Several studies revealed the role of $\mathrm{Hh}$ pathway in tumors; the constitutive activation of $\mathrm{Hh}$ has been observed in cancers, such as lung, stomach, and colon (14). Hitherto, few studies 
focused on the effect of Hh on cigarette-induced airway inflammation. The additional evidence further showed that the Hh pathway could promote the inflammatory process not only in lung diseases but also in other systems. The study by Li et al (15) showed that the inhibition of Hh signaling in rats with arthritis using cyclopamine reduced the expressions of TNF- $\alpha$, IL-1 $\beta$ and IL-6.

Interestingly, the role of $\mathrm{Hh}$ in airway inflammation effectuated via hedgehog interacting protein (HIP), a negative regulator of the Hh pathway, exerts a protective role in COPD pathogenesis (16). HIP competitively binds to all three ligands: Shh, Ihh, and Dhh. Lao et al (17) detected approximately $32 \%$ reduced expression of the HIP gene in human COPD lung tissues as compared to ex-smoker controls with normal lung function. Mice with HIP haploinsufficiency $(H I P+/-)$ are susceptible to present more severe airspace enlargement and emphysema induced by cigarette smoke than the wild-type. Moreover, the study also demonstrated that numbers of lymphoid aggregates, MCP1 (monocyte chemotactic protein 1), MMP-9 and MMP-12 levels increased in $H I P+/$ - mice lung after cigarette smoke exposure. In addition, a significantly increased cell death was detected in lungs from $\mathrm{HIP+/}$ - mice. Our previous study also identified some single nucleotide polymorphisms on a $H I P$ gene associated with the susceptibility of COPD in the Chinese Han population (18).

Consequently, HIP plays a critical role in COPD pathogenesis; reduction in HIP expression probably increases susceptibility to develop emphysema. HIP represses the Hh signaling by competitive binding with three Hh ligands. However, whether the mechanism is linked to the Hh signaling pathway is yet to be elucidated, which has been explored in this study.

In addition, other systems showed that $\mathrm{Hh}$ signaling is critical in T-cell development (19), which plays a significant role in gastric development, homeostasis, and neoplastic transformation (20). Reportedly, Hh signaling components are expressed in acute myeloid leukemia cells (21).

Nevertheless, the current study had some limitations. First, additional experiments are essential to accumulate evidence in order to understand the mechanisme underlying the impact of Hh pathway on inflammatory mediators. Are these inhibitions are recovered by Hh signaling activator? Second, these inflammatory cytokines are also controlled by another transcriptional factor such as NF- $\mathrm{KB}$. Thus, the rela-

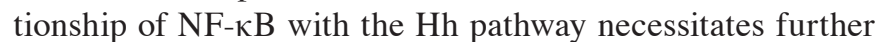
studies. Third, the current study focused on somking-related airway inflammation; however, the impact of Hh signaling on non-smoking-related cases of COPD remains unclear, further studies should be performed.

The current study showed that Hh signaling pathway expressed at a low level in normal alveolar epithelial cells. It can be aberrantly activated by nicotine, resulting in upregulation of certain inflammatory mediators; however, the expression levels of mediators decreased after blockage of the Hh pathway. In conclusion, the Hh pathway might play a major role in cigarette-induced airway inflammation via regulation of inflammatory mediators. Therefore, blocking the Hh signal and diminishing the airway inflammation reaction might be a new therapy of somking-related COPD.

\section{Acknowledgements}

The abstract was presented at the 2017 ERS International Congress on 9th to 13th September 2017 in Milan and published as abstract no. PA4454 in European Respiratory Journal 50 (Suppl 61): 2017 (DOI: 10.1183/1393003.congress-2017).

\section{Funding}

The present study was supported by The Natural Science Foundation of China (grant no. 81500027).

\section{Availability of data and materials}

The datasets used and/or analyzed during the current study are available from the corresponding author on reasonable request.

\section{Authors' contributions}

YG performed ELISA and western blot analysis, and drafted the manuscript. GS, HW and MZ participated in the design of the study and revised the manuscript. All authors read and approved the final manuscript.

\section{Ethics approval and consent to participate}

Not applicable.

\section{Consent for publication}

Not applicable.

\section{Competing interests}

The authors declare that they have no competing interests.

\section{References}

1. McCloskey SC, Patel BD, Hinchliffe SJ, Reid ED, Wareham NJ and Lomas DA: Siblings of patients with severe chronic obstructive pulmonary disease have significant risk of airflow obstruction. Am J Respir Crit Care Med 164: 1419-1424, 2001.

2. Lemjabbar-Alaoui H, Dasari V, Sidhu SS, Mengistab A, Finkbeiner W, Gallup M and Basbaum C: Wnt and hedgehog are critical mediators of cigarette smoke-induced lung cancer. PLoS One 1: e93, 2006.

3. Hanna A and Shevde LA: Hedgehog signaling: Modulation of cancer properies and tumor mircroenvironment. Mol Cancer 15: 24, 2016.

4. Cooper MK, Porter JA, Young KE and Beachy PA: Teratogen-mediated inhibition of target tissue response to Shh signaling. Science 280: 1603-1607, 1998.

5. Kugler MC, Loomis CA, Zhao Z, Cushman JC, Liu L and Munger JS: Sonic hedgehog signaling regulates myofibroblast function during alveolar septum formation in murine postnatal lung. Am J Respir Cell Mol Biol 57: 280-293, 2017.

6. Xu JH, Yang HP, Zhou XD, Wang HJ, Gong L and Tang CL: Autophagy accompanied with bisdemethoxycurcumin-induced apoptosis in non-small cell lung cancer cells. Biomed Environ Sci 28: 105-115, 2015.

7. Bermudez O, Hennen E, Koch I, Lindner M and Eickelberg O: Gli1 mediates lung cancer cell proliferation and sonic hedgehog-dependent mesenchymal cell activation. PLoS One 8: e63226, 2013.

8. Bao J, Ma C, Ran J, Xiong Y, Yan S and Wu L: Wnt/ß-catenin and hedgehog pathways are involved in the inflammatory effect of interleukin 18 on rat chondrocytes. Oncotarget 8: 109962-109972, 2017. 
9. Beachy PA, Karhadkar SS and Berman DM: Tissue repair and stem cell renewal in carcinogenesis. Nature 432: 324-331, 2004.

10. Deng M, Li J, Gan Y, Chen Y and Chen P: Changes in the number of CD31-CD45-Sca-1+ cells and Shh signaling pathway involvement in the lungs of mice with emphysema and relevant effects of acute adenovirus infection. Int J Chron Obstruct Pulmon Dis 12: 861-872, 2017.

11. Fu L, Wu H, Cheng SY, Gao D, Zhang L and Zhao Y: Set7 mediated Gli3 methylation plays a positive role in the activation of sonic hedgehog pathway in mammals. ELife 5: e15690, 2016

12. Liu L, Kugler MC, Loomis CA, Samdani R, Zhao Z, Chen GJ, Brandt JP, Brownell I, Joyner AL, Rom WN and Munger JS Hedgehog signaling in neonatal and adult lung. Am J Respir Cell Mol Biol 48: 703-710, 2013.

13. Peng T, Frank DB, Kadzik RS, Morley MP, Rathi KS, Wang T, Zhou S, Cheng L, Lu MM and Morrisey EE: Hedgehog actively maintains adult lung quiescence and regulates repair and regeneration. Nature 526: 578-582, 2015.

14. Abe Y and Tanaka N: The hedgehog signaling networks in lung cancer: The mechanisms and roles in tumor progression and implications for cancer therapy. Biomed Res Int 2016: 7969286, 2016.

15. Li R, Cai L, Ding J, Hu CM, Wu TN and Hu XY: Inhibition of hedgehog signal pathway by cyclopamine attenuates inflammation and articular cartilage damage in rats with adjuvant-induced arthritis. J Pharm Pharmacol 67: 963-971, 2015.
16. Lao T, Jiang Z, Yun J, Qiu W, Guo F, Huang C, Mancini JD, Gupta K, Laucho-Contreras ME, Naing ZZ, et al: Hhip haploinsufficiency sensitizes mice to age-related emphysema. Proc Natl Acad Sci USA 113: E4681-E4687, 2016.

17. Lao T, Glass K, Qiu W, Polverino F, Gupta K, Morrow J, Mancini JD, Vuong L, Perrella MA, Hersh CP, et al: Haploinsufficiency of hedgehog interacting protein causes increased emphysema induced by cigarette smoke through network rewiring. Genome Med 7: 12, 2015.

18. Guo Y, Gong Y, Pan C, Qian Y, Shi G, Cheng Q, Li Q, Ren L, Weng Q, Chen Y, et al: Association of genetic polymorphisms with chronic obstructive pulmonary disease in the chinese han population: A case-control study. BMC Med Genomics 5: 64-83, 2012.

19. Crompton T, Outram SV and Hager-Theodorides AL: Sonic hedgehog signalling in T-cell development and activation. Nat Rev Immunol 7: 726-735, 2007.

20. Merchant JL and Ding L: Hedgehog signaling links chronic inflammation to gastric cancer precursor lesions. Cell Mol Gastroenterol Hepatol 3: 201-210, 2017.

21. Kakiuchi S, Minami Y, Miyata Y, Mizutani Y, Goto H, Kawamoto S, Yakushijin K, Kurata K, Matsuoka H and Minami H: NANOG Expression as a responsive biomarker during treatment with hedgehog signal inhibitor in acute myeloid leukemia. Int J Mol Sci 18: 486, 2017. 